\title{
NON-FERMI LIQUID STATES OF A GENERAL MODEL OF COPPER-OXIDE METALS
}

\author{
C.M. VARMA
}

Bell Laboratories, Lucent Technologies, Murray Hill, NJ 07974, USA

A summary is given of the minimum requirements posed by experimental results on a theory of copper-oxide metals and of the recent solution of a model of copper-oxygen bonding and anti-bonding bands with the most general two-body interactions allowable by symmetry which satisfies these requirements. Several experiments were proposed to test the theory.

PACS numbers: 74.72.-h, 74.20.Mn, 71.10.Hf, 74.25.-q

\section{Introduction}

Besides their exceptionally high superconducting transition temperatures, copper-oxide $(\mathrm{Cu}-\mathrm{O})$ based metals also have exceptional normal state properties. Landau Fermi-liquid theory and associated quasiparticle concepts, which are a foundation stone for much of our understanding of phenomena in condensed matter appear to be inapplicable to their normal state. The principal problem is the development of a consistent theoretical framework in which the unusual metallic properties can be understood. Moreover, it is necessary to have a theory for the normal state to understand the mechanism for the superconductive instability.

Every transport property in the normal state of copper-oxide metals has a temperature dependence unlike other metals. For example, the electrical resistivity has a linear temperature dependence down to $T_{\mathrm{c}}$ for composition near the highest $T_{\mathrm{c}}$ for any class of $\mathrm{Cu}-\mathrm{O}$ compounds even when that $T_{\mathrm{c}}$ is as low as $10 \mathrm{~K}$. On the other hand, the equilibrium properties, such as specific heat $C_{v}$ and magnetic susceptibility $\chi$ are consistent with the usual temperature dependence and are in fact only about a factor of two enhanced over band structure calculations. The copper-oxide metals are thus qualitatively different from liquid ${ }^{3} \mathrm{He}$ and heavy fermion metals where strong interactions produce strong quantitative renormalizations in both equilibrium and transport properties without changing the asymptotic low temperature dependences and which are properly called Fermi-liquids.

Recent re-examinations [1] of the foundations of Landau's Fermi-liquid theory have confirmed the robustness of the theory for dimensions higher than 1 for any Hamiltonian with non-singular low-energy interactions. Therefore a principal part of the theoretical task is to show that, in a model appropriate for copper-oxide 
metals, elimination of the high energy degrees of freedom leads to a singular effective low energy Hamiltonian. The tremendous variety and number of experiments on copper-oxide metals severely constrain the form of such a low energy Hamiltonian [2].

\section{Constraints from experiments}

A schematic generic phase diagram is drawn in Fig. 1 on the basis of the resistivity data. Where measurements are available every other transport property shows corresponding regions. The insulating-antiferromagnetic phase near $1 / 2$-filling and the superconducting phase are shown in bold lines. The normal state is roughly divided into four regions with dashed lines representing crossovers from one characteristic temperature dependence in transport properties to another. In region 1 the non-Fermi-liquid phase has $\rho(T) \approx \rho_{0}+\rho_{1} T$ and similarly remarkable "simple" anomalies in all the other transport properties. In Bi 2201 resistivity measurements are available from $10 \mathrm{~K}$ to $800 \mathrm{~K}$ and in $\mathrm{La}_{1.85} \mathrm{Sr}_{0.15} \mathrm{CuO}_{4}$ from $40 \mathrm{~K}$ to $800 \mathrm{~K}$. The measured resistivity exponent in both is $1.05 \pm 0.05$. We may safely assume that it is 1 with possible logarithmic corrections. Such a behavior is observed only in a very narrow region near $x_{\mathrm{c}} . \rho(\mathrm{T})$ begins to $d e-$ crease below the linear extrapolation as temperature is decreased in region 3 and is consistent with an asymptotic $T^{2}$ dependence characteristic of a Fermi liquid. On the low doping side, in region 2 , there is a crossover to resistivity increasing with decreasing temperature. This regime may be termed insulating. It is almost certainly related to singular effects of impurity scattering in a non-Fermi liquid. Strictly speaking, one should draw a third axis in Fig. 1 labelled disorder. If the zero-temperature intercept of the high temperature linear resistivity is taken as a

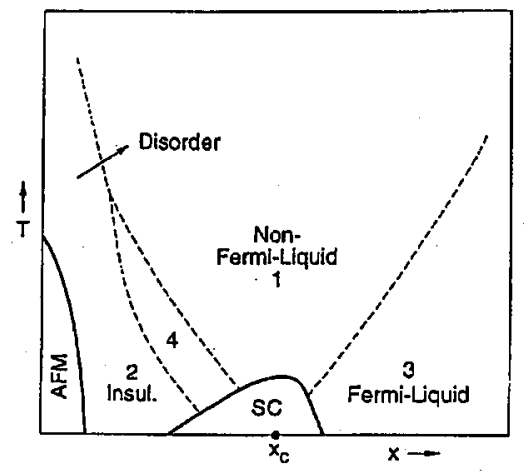

Fig. 1. Schematic generic phase diagram of the quasi-two-dimensional copper-oxide compounds. $x$ is the density of holes doped in the planes. $x_{\mathrm{c}}$ is the "optimum" composition. The antiferromagnetic phase and the superconducting phases, shown inside solid lines, occur through phase transitions. A series of crossovers shown through dashed lines are discussed in the text. The impurity density, as inferred from the extrapolation of the high temperature resistivity to $T=0$, i.e. assuming Mattheisen's rule decreases as $x$ increases. The size of region 4 decreases with increasing disorder for a given $x$. 
measure of disorder one concludes that it generally increases in the available data as $x$ decreases. The limited systematic data with independent variation of disorder and $x$ clearly shows that impurities have a dramatic effect in the underdoped regime while in the overdoped regime, region 3 , their effect is conventional.

The size of the region 4 between region 1 and region 2 on the underdoped side depends upon disorder. The crossover between region 1 and region 4 is marked by a decrease in $C_{v} / T$ and $\chi$ with temperature with $t h$. ir ratio almost independent of temperature [3]. In region 4 the resistivity drops below the linear extrapolation from region 1. This region has been termed the spin-gap region, but this is a misnomer. Not only do magnetic fluctuations, but optical conductivity and Raman scattering intensity in all measured polarizations decrease at low energies from their value in region 1 . In region 2 this decline continues while the resisitivity begins to increase with decreasing temperature.

The schematic phase diagram of Fig. 1 suggests that the anomalous normal state (as well as superconductivity) are controlled by fluctuations around the point $x \approx x_{\mathrm{c}}$ and $T \approx 0$. This is consistent with the marginal Fermi-liquid (MFL) phenomenology [4] which suggests that the breakdown of Landau theory is due to a quantum critical point, QCP (i.e. a singularity at $T=0$ ) near the ideal composition. The normal state anomalies are due to scale invariant fluctuations about the critical point (whose nature was not specified), which itself is inaccessible due to the superconductive instability. The MFL has a single particle self-energy of the form

$$
\Sigma(\omega, q)=\lambda\left(\omega \ln \frac{\omega_{c}}{x}+\mathrm{i} x \operatorname{sgn} \omega\right)
$$

where $x=\max |\omega|$ for $|\omega| \gg T$ and $x=\pi T$ for $T \gg|\omega|, \lambda$ is a coupling constant and $\omega_{\mathrm{c}}$ is a cut-off energy. The quasi-particle renormalization amplitude

$$
z(\omega)=\left(1-\frac{\partial \operatorname{Re} \Sigma}{\partial \omega}\right)^{-1}=\left(1+\lambda \ln \frac{\omega_{\mathrm{c}}}{\mathrm{x}}\right)^{-1}
$$

then vanishes logarithmically as $(\omega, T) \rightarrow 0$.

A microscopic theory should specify the nature of the critical point and the symmetry on either side of it. It should also answer the question: If there is a critical point at $x=x_{\mathrm{c}}, \mathrm{T}=0$, what about its continuation in the $x-T$ plane? Should not there be evidence of nonanalytic properties on a line in the $x-T$ plane? Experimentally, there is indeed a crossover in the properties in the $x-T$ plane from region 1 to region 4 of Fig. 1 . But why a crossover rather than a transition?

Equation (1) gives only the single particle scattering rate $\tau_{\mathrm{sp}}^{-1}$. This was used to understand the observed tunneling conductance $G(V) \sim|V|$ for $T \rightarrow 0$ and to predict the line shapes in single particle spectra. A crucial aspect of the properties of the $\mathrm{Cu}-\mathrm{O}$ metals is that the momentum transport scattering rates, measured in resistivity $\rho(T)$ (as well as in optical conductivity $\sigma(\omega, T)$ and Raman cross-section $S_{\mathrm{R}}(\omega, T)$ ) are also proportional to $\max (|\omega|, T)$. So is the energy scattering rate $\tau_{\mathrm{en}}^{-1}$ measured by thermal conductivity $\kappa(T)$. The experimental result that at $(q, \omega) \rightarrow 0$

$$
\tau_{\mathrm{sp}}^{-1} \sim \tau_{\text {mom }}^{-1} \sim \tau_{\text {en }}^{-1} \sim T
$$

puts a strong constraint on theories. The single particle scattering rate is required in general to be at least as singular as the momentum scattering rate $\tau_{\text {mom }}^{-1}$. Angle 
resolved photoemission should be used to put stricter bounds on the single particle self-energy than have been done so far. But one can be reasonably sure that it is close to (2.1). The electronic specific heat can be calculated from the exact single particle Green function. Equation (2.1) gives $C_{v} \sim N(0) T\left(1+\lambda \ln \omega_{\mathrm{c}} / T\right)$. A more singular self-energy $\operatorname{Re} \Sigma(\omega) \sim \omega^{\alpha}, \alpha<1$ gives $C_{v} \sim N(0) T^{\alpha}$. In the experiments the electronic part of $C_{v}$ is obtained only by subtracting the estimated phonon heat capacity and at $x \approx x_{\mathrm{c}}$ is reported to be consistent with $\sim T$. While logarithmic corrections to it cannot be ruled out, substantial singular departures are ruled out.

The proportionality of the single particle and the transport scattering rates occur if the fluctuations leading to (2.1) are essentially momentum independent (because in that case there are no vertex corrections). But this poses the serious dilemma that on the one hand we wish to be near a critical point, on the other that we need (nearly) momentum independent fluctuations.

The trivial way to get (2.3) is if the experiments are in a temperature range $T \omega^{*}$ where $\omega^{*}$ is the characteristic frequency of some fluctuations which scatter the fermions. Then the density of such fluctuations is $\sim T$ giving (2.3). This is ruled out by the specific heat (and magnetic susceptibility) experiments. If such fluctuations are collective modes like spin or density fluctuations of fermions, a characteristic enhancement in $\gamma=C_{v} / T$ of $\mathrm{O}\left(E_{\mathrm{F}} / \omega^{*}\right) \sim \mathrm{O}\left(10^{2}\right)$ must occur as in the heavy fermion compounds. Experimentally, the specific heat and magnetic susceptibility at the ideal composition are consistent with Fermi-liquid behavior, $\sim T$ and constant respectively, with no more than about a factor of 2 enhancements over non-interacting electrons in the measured temperature range. (The data however does ailow for logarithmic or small power law corrections.) This as well as the fact that $\sigma(\omega, T)$ and $S_{\mathrm{R}}(\omega, T)$ behaves smoothly in the range $\omega T \omega_{\mathrm{c}}$ where $\omega_{\mathrm{c}} \sim \mathrm{O}\left(\frac{1}{2} \mathrm{eV}\right)$ suggests that there is no low-energy scale near optimum doping and that the upper cut-off frequency of the fluctuations is very high, $\mathrm{O}\left(\frac{1}{2} \mathrm{eV}\right)$.

It is hard to imagine that the fluctuations due to the antiferromagnetic $T=0$ critical point at $x \approx 0.02$ can have much to do with phenomena at $x \approx x_{\mathrm{c}}$, with $x_{\mathrm{c}}=0.15$. Temperature independent magnetic correlation lengths of about $2 \AA$ are observed at $x \approx x_{\mathrm{c}}$ in $\mathrm{YBa}_{2} \mathrm{Cu}_{3} \mathrm{O}_{6.93}$ [5]. Temperature-dependent lengths of $\approx 20 \AA$ at $x \approx x_{\mathrm{c}}$ in $\mathrm{La}_{1.85} \mathrm{Sr}_{0.15} \mathrm{CuO}_{4}$ [6] but with less than $\approx 10 \%$ of the total frequency integrated spectral weight in the $q$-dependent part. The normal state anomalies are identical in both compounds. Similarly, there is no evidence of any universal phase-separation fluctuations or charge density fluctuations in different compounds at $x \approx x_{\mathrm{c}}$. It would appear that if a critical point is responsible for the unusual metallic state, it must be associated with some quite unusual order parameter which is hard to detect.

Equations (1.1) and (1.3) cannot be used to understand the observed anomalies in NMR [7], Hall effect [8], and magnetoresistance [9]. The anomalies in the Hall conductivity, $\sigma_{x y}$ and magnetoresistance are quite revealing. In $\mathrm{YBa}_{2} \mathrm{Cu}_{3} \mathrm{O}_{6.9}$, where band-structure calculations give a very small usual Hall conductivity due to particle-hole symmetry, the Hall angle $\Theta_{\mathrm{H}}=\sigma_{x y} / \sigma_{x x}$, with magnetic field perpendicular to the plane, varies approximately as $T^{-2}$ between $100 \mathrm{~K}$ and $300 \mathrm{~K}$. In the same range the normalized magnetoresistance $\Delta \rho(H) / \rho$ varies roughly as $T^{-4}$ with $\Theta_{\mathrm{H}}^{2} / \Delta \rho / \rho \approx O(1)$. Kotliar et al. [10] have found the observed behavior 
in a solution of the Boltzmann equation which besides a scattering rate $\tau_{\mathrm{tr}}^{-1} \sim T$, contains a phenomenological skew scattering rate proportional to the applied magnetic field which is $\sim T^{-1}$.

Perhaps the most astonishing of the normal state anomalies are the nuclear relaxation rates ${ }^{\mathrm{Cu}} T_{1}^{-1}$ and ${ }^{\mathrm{O}} T_{1}^{-1}$ of copper and oxygen nuclei respectively [7]. While $\left({ }^{\mathrm{Cu}} T_{1} T\right)^{-1}$ appears to diverge as temperature is decreased suggesting singular local magnetic fluctuations at the $\mathrm{O}$ nuclei, $\left({ }^{\circ} T_{1} T\right)^{-1}$ is a constant, which is the conventional behavior. Furthermore, $\left({ }^{\circ} T_{1} T K\right)^{-1}$, where $K$ is the measured Knight shift at oxygen, is within $20 \%$ a constant, irrespective of the compound studied or the density $x$ in any given compound in the metallic range. Other experiments show that copper and oxygen orbitals are well hybridized. Nevertheless, it appears that the local magnetic fluctuations at copper and oxygen sites are quite different.

It is axiomatic that the fluctuations responsible for the anomalous metallic state also are responsible for the instability to the superconducting state. The anomalous fluctuations develop a gap in the superconducting state as predicted [11] and observed in a wide variety of experiments on quasiparticle relaxation rate deduced through transport experiments [12] and in angle resolved photoemission experiments [13]. The symmetry of the superconducting state appears to be consistent with " $D$-wave" (if the lattice is assumed tetragonal) [14]. This issue is not completely settled yet [15].

To summarize, existing experiments require an internally consistent microscopic theory to:

(i) Reproduce the phase diagram of Fig. 1 with a non-Fermi-liquid metallic phase near the composition for the highest $T_{\mathrm{c}}$ with crossovers to Fermi liquid on the high doping side and with a strong tendency to insulating behavior due to disorder in the underdoped regime. The underdoped regime shows loss at low energies of both particle-hole excitations (in spin as well as charge channels) and of single particle excitations.

(ii) Have equilibrium properties like specific heat and magnetic susceptibility near the ideal composition consistent in the measured range of $T$ with characteristic Fermi-liquid behavior to within small corrections and in magnitude be within factors of $\mathrm{O}(2)$ of those for non-interacting electrons.

(iii) Ilave long wavelength transport relaxation rates used to interpret electrical conductivity and thermal conductivity at the ideal composition which satisfy Eq. (3). At $x=x_{\mathrm{c}}$ the fluctuations leading to the anomalous transport should have no scale other than a cut-off of the $\mathrm{O}(1 / 2 \mathrm{eV})$.

(iv) The fluctuations in metallic state should be such that in the presence of a magnetic field, they acquire additional singularities. This appears necessary to understand the anomalous Hall effect and magnetoresistance experiments.

(v) The fluctuations should have a symmetry such that they produce singular local magnetic fluctuations at copper nuclei to give the observed anomalies in the copper nuclei relaxation rate, but no singular local magnetic fluctuations at the oxygen nuclei. 
(vi) The fluctuations should be capable of producing a pairing instability of $D$-wave symmetry.

There are of course many other special properties discovered in a subject in which $\mathrm{O}\left(5 \times 10^{4}\right)$ papers have been published. But we regard the requirements listed above as the most basic or a least the irreducible minimum.

\section{Choice of a model}

The choice of a model with which to do microscopic theory should be influenced by the fact that copper-oxide metals are unique. None of the thousands of transition metal compounds studied share their properties. The point of view taken here and elsewhere [16] is that the unique properties of $\mathrm{Cu}-\mathrm{O}$ metals arise from their unique chemistry in which ionic interactions play a crucial dynamical role. The divalent transition metal oxides at $1 / 2$-filling can be put on the diagram [17] in Fig. 2 in which one of the axes is the normalized local repulsion energy $U$ on the transition metal (TM)

$$
U / W=\left[E(\mathrm{TM})^{3+}+E(\mathrm{TM})^{1+}-2 E(\mathrm{TM})^{2+}\right] / W,
$$

where $W$ is the bandwidth. The other axis is the ionic energy

$$
E_{x} / W=\left[E\left(\mathrm{TM}^{1+} \mathrm{O}^{1-}\right)-E\left(\mathrm{TM}^{2+} \mathrm{O}^{2-}\right)\right] / W .
$$

$U$ is the energy to convert two transition metal ions with formal charge state $2^{+}$ to one with formal charge state $1^{+}$and the other to $3^{+}$, while $E_{x}$ is the energy to transfer charge from the ground state configuration of a transition metal ion with charge $2^{+}$and a nearest neighbor oxygen ion with $2^{-}$to transition metal ion with

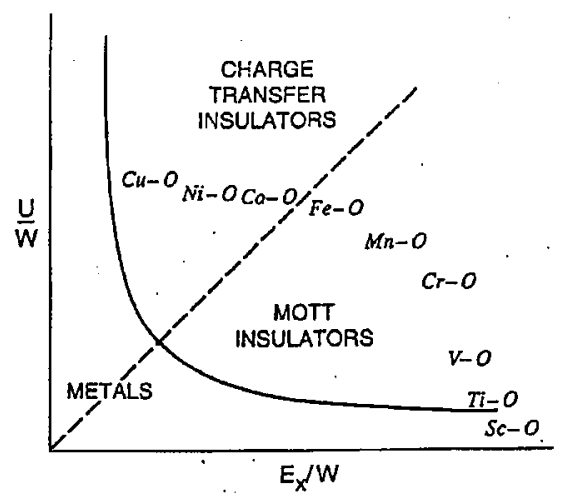

Fig. 2. The Zaanen-Sawatzky-Allen (ZSA) phase diagram for $3 d$-transition metal oxides, slightly modified and showing the schematic change in the position of the transition metal oxides going from left to right of the periodic table. The modification is that one of the axes is the ionic energy $E_{x}$ defined through particle-hole spectra. ZSA used the charge transfer gap $\Delta$ defined through one-particle spectra. $E_{x}<\Delta$ due to particle-hole interactions. $E_{x} / W$ is the more appropriate parameter to characterize the metal-insulator transition than $\Delta / W$. 
charge $1^{+}$and oxygen ion with charge $1^{-}$. Screening and dipole corrections, etc. in the solid are included in the definitions of $U$ and $E_{x}$.

As one moves from the left to the right of the periodic table, the ionization energy of the TM falls thereby decreasing $E_{x} / W$ relative to $U / W$ with corresponding movement in Fig. 2. In the insulating state of $\mathrm{Cu}-\mathrm{O}, E_{x}$ is only about $1 \mathrm{eV}$. It is a charge-transfer insulator with the lowest energy one-particle spectra primarily on copper, $\mathrm{Cu}^{2+} \rightarrow \mathrm{Cu}^{+}$while the one-hole spectra is primarily in oxygen, $\mathrm{O}^{2-} \rightarrow \mathrm{O}^{1-}$. In the metallic state, obtained by doping, there are charge fluctuations on copper and on oxygen of similar magnitude. $E_{x} \approx 1 \mathrm{eV}$ is made up of two sets of energies, the atomic level energies and the Madelung or ionic energies each of which is $\mathrm{O}(10 \mathrm{eV})$. Indeed, all transition metal oxides owe their structural stability to the ionic energies. But in the metallic state these energies have little dynamical role to play in most TM-oxides because there are hardly any fluctuations on oxygen, i.e. although the ionic fluctuation energy term in the Hamiltonian

$$
\sum_{i<j} V_{i j} n_{i} n_{j}
$$

has $\sum_{i<j} V_{i j} \sim \mathrm{O}(10 \mathrm{eV})$, the fluctuation $\delta n_{0 j}$ on the oxygen ions require a large energy and are insignificant. An effective low-energy Hamiltonian of the Hubbard form is then adequate. This is not true in the metallic state of $\mathrm{Cu}-\mathrm{O}$ where $\left\langle\delta n_{0}\right\rangle /\left\langle n_{0}\right\rangle \sim \mathrm{O}(1)$.

One of the aims of this paper is to show that long-range interactions, if sufficiently strong, lead to qualitatively new features in the phase diagram of the model.

\section{Summary of the properties of the model}

We find that it is enough to consider a two-band model representing $\mathrm{Cu}$ and $O$ bonding $b$ and anti-bonding $a$, bands. In the hole representation, the chemical potential $\mu$ is in the lower band as shown. For non-interacting electrons $\mu$ would be in the middle of band $a$ at $1 / 2$-filling as in $\mathrm{La}_{2} \mathrm{CuO}_{4}$ or $\mathrm{YBa}_{2} \mathrm{Cu}_{3} \mathrm{O}_{6}$. For hole doping, as for most $\mathrm{Cu}-\mathrm{O}$ compounds, $\mu$ rises with doping $x$, for example in $\mathrm{La}_{2-x} \mathrm{Sr}_{x} \mathrm{CuO}_{4}$ or $\mathrm{YBa}_{2} \mathrm{Cu}_{3} \mathrm{O}_{6+x}$. We find it important to consider the most general form of two-body interactions allowable by symmetry in the space of these two bands. When the strength of the interactions is on the same scale as the overall electronic bandwidth every term has a crucial role to play.

If $\mu$ were in the gap between bands $a$ and $b$, the model is identical to the excitonic insulator problem [18], which has been extensively discussed. We investigate here the model with $\mu$ in one of the bands. This changes the problem substantively.

It is well known that interactions completely alter the one-electron picture at 1/2-filling; a gap develops around the chemical potential and the relative amount of $\mathrm{Cu}$ and $\mathrm{O}$ character of the occupied and unoccupied states is drastically altered. $\mu$ stays in the band significantly away from $1 / 2$-filling but the relative $\mathrm{Cu}-\mathrm{O}$ character of the occupied and unoccupied states is again expected to be quite different from the one-electron picture. If no change in the lattice symmetry occurs due to the interactions, this is formally describable by a $q=0$ instability of the one-electron 
band structure to a state in which

$$
T_{x} \sim \operatorname{Re} \sum_{k} \mathcal{F}_{x}(k)\left\langle a_{k \sigma}^{+} b_{k \sigma}\right\rangle \neq 0 .
$$

Here $\mathcal{F}_{x}(k)$ is a form factor expressing the relative $\mathrm{Cu}$ and $\mathrm{O}$ character of states in bands $a$ and $b$. We will see that $T_{x}$ is closely related to the relative average charge in $\mathrm{Cu}$ and $\mathrm{O}$ orbitals. The mean-field free energy as a function of $T_{x}$ is shown in Fig. 3a. With $T_{x} \neq 0$, the orbitals must be rehybridized leading to new bands $\alpha$ and $\beta$ of the same general form as $a$ and $b$. This is expected to occur for strong interactions at very high temperatures for all $x$ of interest just as it does at $x=0$. For the most general interactions, such a transition is of first order, as for a free energy of the form shown in Fig. 3a. Previous investigations $[19,20]$ of the model had focused on this charge transfer instability.
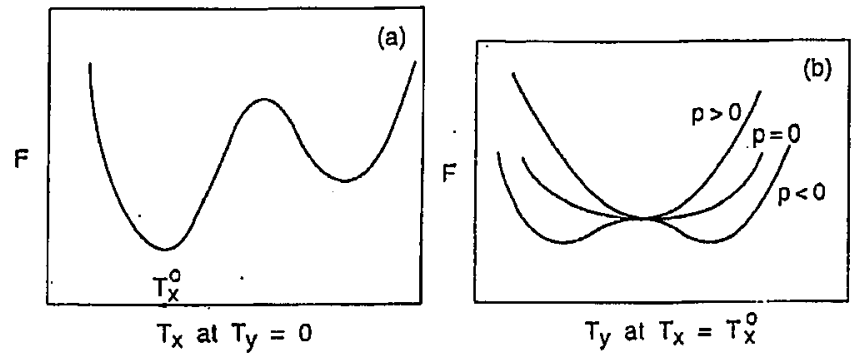

Fig. 3. (a) The calculated mean-field free energy as a function of the real part of interband order parameter $T_{x}$ described in the text. (b) For a fixed $T_{x}=T_{x}^{0}$, the free energy as a function of the imaginary part of the interband order parameter $T_{y}, T_{y}$ takes the value 0 for $p>0$ and a finite value below $p<0$ through a second-order transition $p$ is a parameter, defined in terms of the parameters of the Hamiltonian and for a given compound can be varied by varying the electron density, temperature or pressure. $T_{y} \neq 0$ corresponds to a circulating current pattern in the ground state shown in Fig. 4.

We show that the model also has an intercsting second-order transition in the Ising class to a state in which

$$
T_{y} \sim \operatorname{Im} \sum_{k \sigma} \mathcal{F}_{y}(k)\left\langle a_{k \sigma}^{+} b_{k \sigma}\right\rangle \neq 0 .
$$

The mean-field free energy, for a fixed $T_{x} \neq 0$, as a function of $T_{y}$ for various values of a parameter $p$ which is a function of $x$ and $T$ is shown in Fig. 3b. This transition therefore occurs on a line $T_{c}(x)$ in the $x-T$ plane. We will be specially interested in the properties in the vicinity of the point $x=x_{\mathrm{c}}(0)$ where $T_{\mathrm{c}}=0$ which we identify as the quantum critical point.

A finite $T_{y}$ provides an additional relative phase to the wave functions at $\mathrm{Cu}$ and $\mathrm{O}$ sites in a unit cell. We find that the ground state with a finite $T_{y}$ corresponds to a fourfold pattern of circulating current within a unit cell with all cells staying equivalent. This is illustrated in Fig. 4 . We may call it the circulating current (CC) phase. We discuss such a phase in the metallic state. This leads to a 


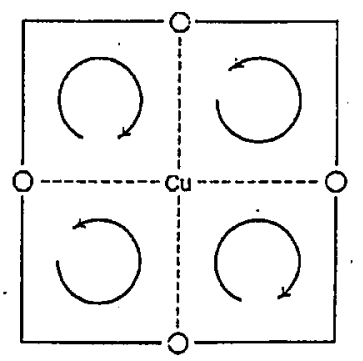

Fig. 4. The deduced ground state current distribution pattern in a unit cell in the circulating current phase.

very special nature of the collective fluctuations near the QCP due to scattering of the fluctuations by low-energy particle-hole excitations at the Fermi surface. The form of the fluctuations is in the extreme quantum limit where the logarithm of the frequency scales with the momentum dependence, i.e. the QCP has dynamical critical exponent $z_{\mathrm{d}}=\infty$.

The low-energy Hamiltonian for the model is derived and analyzed and it is shown that a systematic and controlled analysis is possible. The physical properties of the pure model have been investigated in and a beginning is made to consider the effect of impurities in the properties of the model. Impurities are strongly relevant near a $z_{\mathrm{d}}=\infty$ transition. For arbitrarily small concentration, they convert the line $T_{c}(x)$ to a crossover. We also show that for arbitrarily small concentration of impurities in a non-Fermi liquid, the Fermi surface withers away at low temperatures. The density of states at the chemical potential and the resistivity at $T \rightarrow 0$ is infinity (unless superconductivity intervenes).

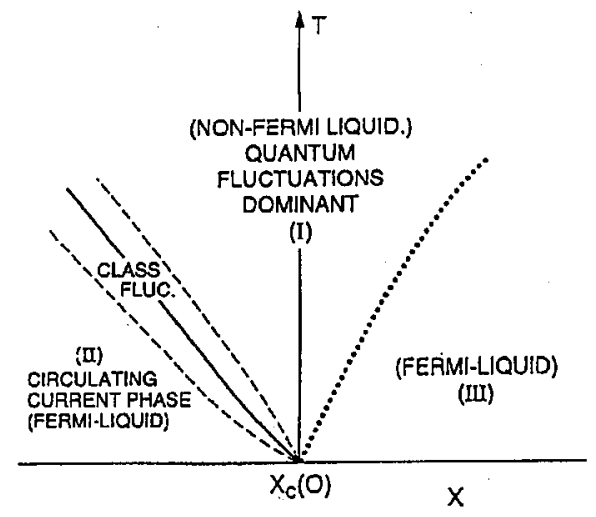

Fig. 5. The theoretical phase diagram in the pure limit. 
Not including the effect of disorder the schematic deduced phase diagram of the model is shown in Fig. 5. In region I, the properties are determined by quantum fluctuations and are that of a marginal Fermi liquid with a crossover to a Fermi-liquid regime in region III. In region II $T_{y} \neq 0$ in the pure limit. This phase should have Fermi-liquid properties at low temperatures in the pure limit but with different parameters from that of region III. The transition between regions I and II turns into a crossover at arbitrarily small concentration of impurities. At low temperatures impurities are expected to lead to a further crossover in region III to an insulating regime with zero density of states at the chemical potential. Antiferromagnetism phase near $1 / 2$-filling and superconductivity phase near $x_{\mathrm{c}}$ which are also properties of the model are not shown.

A study of the pairing instability due to the exchange of the circulating current fluctuations has been made and a propensity towards a " $D$-wave" superconducting instability is indicated.

A paper with detailed calculations has been prepared [21]. The application to NMR is being published separately [22].

\section{References}

[1] R. Shankar, Rev. Mod. Phys. 66, 129 (1994); C. Castellani, C. DiCastro, W. Metzner, Phys. Rev. Lett. 72, 3161 (1994); A. Houghton, J.B. Marston, Phys. Rev. 48, 7790 (1993); A.H. Castro Neto, E. Fradkin, Phys. Rev. Lett. 72, 1393 (1994).

[2] (a) The most complete references to the experimental results are Physica C 185-189 (1991) reprinted; Physica $C$ 235-240 (1994) reprinted. (b) A source of excellent review articles is the series Physical Properties of High Temperature Superconductors, Ed. D.M. Ginsburg, World Scientific, Singapore, Vol. I (1989), Vol. II (1990), Vol. III (1992), Vol. IV (1994).

[3] J.W. Loram, K.A. Mirza, J.M. Wade, J.R. Cooper, W.Y. Liang, Physica $C$ 235, 134 (1994).

[4] C.M. Varma, P.B. Littlewood, S. Schmitt-Rink, E. Abrahams, A.E. Ruckenstein, Phys. Rev. Lett. 63, 1996 (1989); G. Kotliar, E. Abrahams, A.E. Ruckenstein, C.M. Varma, P.B. Littlewood, S. Schmitt-Rink, Europhys. Lett. 15, 655 (1991); (a) P.B. Littlewood, C.M. Varma, J. Appl. Phys. 69, 4979 (1991); C. Berthier, J.A. Gillet, T. Auler, Y. Berthier, M. Horvatic, P. Segranson, J.Y. Henry, Phys. Scr. Vol. T 49, 131 (1993).

[5] J.M. Tranquada, P.M. Gehring, G. Shirane, S. Shamoto, M. Sat, Phys. Rev. B 46, 5561 (1994); J. Rossat-Mignod, L.P. Regnault, P. Bourges, P. Burlet, C. Vetti, J.Y. Henry, Physica B 192, 109 (1993); L.P. Regnault, P. Bourges, P. Burlet, J.Y. Henry, J. Rossat-Mignod, Y. Sidis, C. Vettier, Physica C 235, 59 (1994).

[6] T.E. Mason, G. Aeppli, S.M. Hayden, A.P. Ramirez, H.A. Mook, Phys. Rev. Lett. 71, 919 (1993); S.M. Hayden, G. Aeppli, H.A. Mook, T.G. Perring, T.E. Mason, S.-W. Cheong, Z. Fisk, Phys. Rev. Lett. 76, 1344 (1996).

[7] An excellent review article is C.H. Pennington, C.P. Slichter, Vol. III of Ref. [2b].

[8] (a) T.R. Chien, Z.Z. Wang, N.P. Ong, Phys. Rev. Lett. 67, 2089 (1991); J.M. Harris, Y.F. Yan, N.P. Ong, Phys. Rev. B 46, 14293 (1992). (b) H.Y. Hwang, B. Batlog, H. Takagi, H.L. Kao, J. Kwo, R.J. Cava, J.J. Krajewski, W.F. Peck Jr., Phys. Rev. Lett. 72, 2636 (1994). 
[9] J.M. Harris, Y.F. Yan, P. Matl, N.P. Ong, P.W. Anderson, T. Kimura, K. Kitazawa, Phys. Rev. Lett. 75, 1391 (1995).

[10] G. Kotliar, A. Sengupta, C.M. Varma, Phys. Rev. 53, 3573 (1996).

[11] Y. Kuroda, C.M. Varma, Phys. Rev. 42, 8619 (1990) and Ref. [4a].

[12] M.C. Nuss, P.M. Mankiewich, M.L. O'Malley, E.H. Westerwick, P.B. Littlewood, Phys. Rev. Lett. 66, 3305 (1991).

[13] See for example, H. Ding, J.C. Campuzano, A.F. Bellman, T. Yokoya, M.R. Norman, M. Randeira, T. Takahashi, H. Katayama-Yoshida, T. Mochiku, K. Kadowaki, G. Jennings, Phys. Rev. Lett. 74, 2784 (1995).

[14] D.J. Van Harlingen, Rev. Mod. Phys. 67, 515 (1995); W.N. Hardy, D.A. Bonn, D.C. Morgan, R. Liang, K. Zhang, Phys. Rev. Lett. 70, 3999 (1993); C.C. Tsuei, J.R. Kirtley, C.C. Chi, Lock See Yu-Jahnes, A. Gupta, T. Shaw, J.Z. Sun, M.B. Ketchen, Phys. Rev. Lett. 73, 593 (1994).

[15] A.G. Sun, D.A. Gajewski, M.B. Maple, R.C. Dynes, Phys. Rev. Lett. 72, 2267 (1994).

[16] C.M. Varma, S. Schmitt-Rink, E. Abrahams, Solid State Commun. 62, 681 (1987), and in Novel Mechanisms of Superconductivity, Eds. V. Kresin, S. Wolf, Plenum Press, New York 1987. A model with both $\mathrm{Cu}$ and $\mathrm{O}$ orbitals was proposed at the same time by V. Emery, Phys. Rev. Lett. 58, 2794 (1987). But the ionic interactions played no role in that model.

[17] J. Zaanen, G.A. Sawatsky, J.W. Allen, Phys. Rev. Lett. 55, 418 (1985).

[18] L.V. Keldysh, Yu.V. Kopaev, Sov. Phys.-Solid State 6, 2219 (1965); J. des Cloizeaux, J. Phys. Chem. Solids 26, 259 (1965); D. Jerome, T.M. Rice, W. Kohn, Phys. Rev. 158, 462 (1967).

[19] P.B. Littlewood, C.M. Varma, S. Schmitt-Rink, E. Abrahams, Phys. Rev. Lett. 63, 2602 (1989); P.B. Littlewood, Phys. Rev. B 42, 10075 (1990); Y. Bang, G. Kotliar, R. Raimondi, C. Castelli, M. Grilli, Phys. Rev. B 47, 3323 (1993); S. Coppersmith, P.B. Littlewood, Phys. Rev. B 42, 3966 (1990); R. Putz, B. Ehlers, L. Lilly, A. Muramatsu, W. Hanke, Phys. Rev. B 41, 853 (1990).

[20] M. Grilli, R. Raimondi, C. Castelli, C. Di Castro, G. Kotliar, Phys. Rev. Lett. 67, 259 (1991), Phys. Rev. B 47, 3331 (1993); J.C. Hickes, A.E. Ruckenstein, S. Schmitt-Rink, Phys. Rev. B 45, 8185 (1992).

[21] C.M. Varma, Phys. Rev. B, submitted, preprint available on http:/www.lanl.gov condmat/9607105.

[22] C.M. Varma, Phys. Rev. Lett. 77, 3431 (1996). 